\title{
Nuclear Magnetic Relaxation by the Manganese in Aqueous Suspensions of Chloroplasts ${ }^{\dagger}$
}

\author{
Thomas J. Wydrzynski, ${ }^{\ddagger}$ Stephen B. Marks, Paul G. Schmidt, ${ }^{\S}$ Govindjee, ${ }^{\natural}$ and Herbert S. Gutowsky*
}

ABSTRACT: Proton and oxygen-17 NMR relaxation rate $\left(T_{1}^{-1}\right.$ and $\left.T_{2}^{-1}\right)$ data are presented for aqueous suspensions of dark-adapted chloroplasts. It is concluded from the dependence of the proton relaxation rates (PRR) upon Mn concentration that $T_{1}^{-1}$ and $T_{2}^{-1}$ are determined largely by the loosely bound Mn present in the chloroplast membranes. The frequency and temperature dependences of the PRR are characteristic of $\mathrm{Mn}$ (II). The effects of oxidants (e.g., ferricyanide) and reductants (e.g., tetraphenylboron) on the PRR indicate that only about one-third to one-fourth of the loosely bound $\mathrm{Mn}$ is present in the dark-adapted chloroplasts as
$\mathrm{Mn}(\mathrm{II})$, the remainder being in a higher oxidation state(s), probably Mn(III). The frequency dependence of the PRR for the chloroplast suspensions was fitted by a simplified form of the Solomon-Bloembergen-Morgan equations, and the following parameters were obtained: $\tau_{\mathrm{S}}=(1.1 \pm 0.1) \times 10^{-8} \mathrm{~s}$; $\tau_{\mathrm{M}}=(2.2 \pm 0.2) \times 10^{-8} \mathrm{~s} ;$ and $B=(0.9 \pm 0.09) \times 10^{19}$. The oxygen-17 $T_{1}$ and $T_{2}$ data for suspensions before and after treatment with a detergent are consistent with the location of the manganese in the interior of the thylakoids. An analysis of the relaxation rates shows that the average lifetime of a water molecule inside a thylakoid is $>1 \mathrm{~ms}$.
1 he involvement of manganese ions in photosynthesis was first suggested in the early studies of Pirson (1937). Much later, the site of its activity was assigned to the oxygen-evolving mechanism of photosystem II (Kessler et al., 1957; Spence and Possingham, 1961; Cheniae and Martin, 1966; Homann, 1968). Subsequently, Kok et al. (1970) proposed a model for oxygen evolution in which a photochemically activated intermediate accumulates up to 4 oxidizing equiv before reacting with water to produce oxygen. Manganese can exist in several oxidation states and thus it could play a central role in the charge accumulation (Cheniae, 1970; Olson, 1970; Renger, 1970; Earley, 1973). However, until recently there has been no direct experimental evidence to show that manganese changes its oxidation state during photosynthesis (Wydrzynski et al., 1976a,b).

The current view is that manganese is complexed to some protein bound to the chloroplast membrane. However, attempts to isolate a manganese protein and to reconstitute oxygen evolution have failed (Cheniae and Martin, 1966; Lagoutte and Duraton, 1975; Henriques and Park, 1976). One possible exception is the stimulation of oxygen evolution by a low-molecular-weight manganese polypeptide isolated from Phormidium luridium (Tel-Or and Avron, 1974). In any event, if one wishes to study the role of manganese in oxygen evolution, it is necessary to use an experimental approach which responds to changes in situ of the manganese.

The unpaired electrons of $\mathrm{Mn}$ (II) can, in favorable instances, give rise to a detectable electron paramagnetic reso-

\footnotetext{
${ }^{\dagger}$ From the Departments of Physiology and Biophysics, Botany, and Chemistry, University of Illinois, Urbana, Illinois 61801. Received November 16, 1977. Supported by research grants from the National Science Foundation to Govindjee (PCM 76-11657) and to H. S. Gutowsky (MPS 73-0498 and CHE 77-04585) and from the United States Public Health Service to P. G. Schmidt (NIH GM 18038).

$\ddagger$ Trainee of United States Public Health Service Training Grant in Cellular and Molecular Biology, GM 7283-1, Sub Program 604. Present address: Department of Chemistry, University of California, Berkeley, Calif. 94720.

$\$$ Present address: OkJahoma Medical Research Foundation, Oklahoma City, Oklahoma 73104.

TMailing address: Department of Botany, University of Illinois, Urbana, Ill. 61801 .
}

nance $\left(E R^{1}\right)$ spectrum. However, so far there has been only one report of EPR spectra from $\mathrm{Mn}$ bound to chloroplasts (Siderer et al., 1977). Another possibility is that neighboring magnetic nuclei are perturbed enough by the large magnetic moment of the unpaired electrons in $\mathrm{Mn}$ (II) to be sensitive to changes in its oxidation state. Mn(II) usually maintains some water ligands even when bound to large macromolecules and the electronic relaxation of $\mathrm{Mn}$ (II) is such that the relaxation rates of the protons in the solvation sphere are strongly enhanced. Furthermore, all of the water protons share in the enhancement because of the fast proton exchange between solvation sphere and solvent. For these reasons, it seemed likely to us that the proton relaxation rates (PRR) of the water could be used to detect changes in situ of the $\mathrm{Mn}$ in aqueous suspensions of chloroplasts.

Preliminary experiments (Wydrzynski et al., 1975) showed that treatments which affect the amount of bound manganese in chloroplast membranes have a large effect on the spin-lattice or longitudinal relaxation rate $\left(T_{1}^{-1}\right)$ of the water protons. This included washing the membranes with alkaline tris(hydroxymethyl)aminomethane (Tris) buffer or $\mathrm{NH}_{2} \mathrm{OH}$-ethylenediaminetetraacetic acid (EDTA) solution. Also, the addition of oxidants led to a decrease in $T_{1}^{-1}$, whereas the addition of reductants caused a large increase, suggesting that the PRR could be used to detect changes in the oxidation state. In a second set of experiments (Wydrzynski et al., 1976a), the aqueous suspensions were exposed to a series of intense light flashes after $n$ of which $(n=0,1,2, \ldots 20)$ the proton transverse (spin-spin) relaxation rate $\left(T_{2}^{-1}\right)$ was measured. The response of $T_{2}^{-1}$ to light flashes is very similar to that of oxygen evolution, both sequences exhibiting a damped oscillation with a periodicity of four flashes.

Thus, the proton relaxation rates are closely associated with the charge-accumulating mechanism for oxygen evolution by

\footnotetext{
${ }^{1}$ Abbreviations used are: PRR, proton relaxation rates (i.e., $T_{1}{ }^{-1}$ and $T_{2}{ }^{-1}$ ); EPR, electron paramagnetic resonance; Tris, tris(hydroxymethyl)aminomethane; EDTA, ethylenediaminetetraacetic acid; Hepes, $N$-2-hydroxyethylpiperazine- $N^{\prime}$-2-ethanesulfonic acid; chl, chlorophyll; FID, free induction decay; SBM, Solomon, Bloembergen, and Morgan; chlpts, chloroplasts; T-A chlpts, Tris-acetone washed chloroplasts; $\mathrm{Ph}_{4} \mathrm{~B}$ tetraphenylboron; $\mathrm{FeCy}$, potassium ferricyanide, $\mathrm{K}_{3} \mathrm{Fe}(\mathrm{CN})_{6}$.
} 
photosystem II. The purpose of the present study was to determine whether the enhanced rates found in aqueous suspensions of chloroplasts, compared to buffered suspensions without chloroplasts, are indeed caused primarily by $\mathrm{Mn}(\mathrm{II})$. Several different approaches were used. The total $\mathrm{Mn}$ concentration of the chloroplasts was varied, measured, and compared with the proton relaxation rates. The frequency dependence of the proton relaxation was observed for the suspensions and compared with that reported for known $\mathrm{Mn}$ (II) systems. Also, the effects of redox agents upon the frequency dependence were studied. And, finally, some measurements of ${ }^{17} \mathrm{O}$ relaxation were made for suspensions in enriched water.

\section{Experimental Procedure}

Sample Preparation. The chloroplasts were obtained from dwarf peas (Pisum sativum, var, progress no. 9). The seeds were soaked in aerated deionized water for $12 \mathrm{~h}$ and planted in vermiculite. At planting, each flat received $1 \mathrm{~L}$ of Hoagland's solution (Johri and Varner, 1964) containing $9 \mu \mathrm{M}$ $\mathrm{MnCl}_{2}$; thereafter, the plants received only deionized water. The plants were grown under a 16 -h light $\left(23^{\circ} \mathrm{C}\right) / 8$-h dark cycle $\left(20^{\circ} \mathrm{C}\right.$ ). Light intensity was $15 \mathrm{~W} / \mathrm{m}^{2}$ (ITT F72512/cool white fluorescence lamps).

Chloroplasts were isolated from 12-16 day old plants. Leaves were homogenized in a Waring blender for $\sim 15 \mathrm{~s}$ in a medium containing $50 \mathrm{mM} \mathrm{N}$-2-hydroxyethylpiperazine$N^{\prime}$-2-ethanesulfonic acid (Hepes) buffer, adjusted to $\mathrm{pH} 7.5$ with $\mathrm{NaOH}, 400 \mathrm{mM}$ sucrose, and $10 \mathrm{mM} \mathrm{NaCl}$. Also, the homogenization medium included $0.5 \%$ bovine serum albumin along with $10 \mathrm{mM}$ sodium ascorbate. The homogenate was filtered through four layers of cheesecloth and a $10 \mu \mathrm{m}$ mesh nylon cloth, centrifuged, and finally resuspended in the complete medium. Chlorophyll was determined by the method of Arnon (1949), and the suspensions were adjusted to a final concentration of $3 \mathrm{mg}$ of chlorophyll $(\mathrm{chl}) / \mathrm{mL}$.

Manganese was extracted from the chloroplast membranes either by magnesium exchange according to the procedure of Chen and Wang (1974) or by washing in Tris-acetone according to the procedure of Yamashita and Tomita (1974). For extraction by magnesium exchange, chloroplasts were incubated in the isolation media containing $\mathrm{MgCl}_{2}$ at various $[\mathrm{Mg}] /[\mathrm{chl}]$ ratios $(0 ; 167 ; 332 ; 667 ; 2500 ; 10000)$ for $2 \mathrm{~h}$ at $4{ }^{\circ} \mathrm{C}$. The chloroplasts were then centrifuged and resuspended in the isolation medium to a final concentration of $3 \mathrm{mg}$ of $\mathrm{chl} / \mathrm{mL}$. For Tris-acetone extraction the chloroplasts were suspended in $0.8 \mathrm{M}$ Tris buffer, $\mathrm{pH} 8.2$, containing $20 \%$ acetone at $200 \mu \mathrm{g}$ of $\mathrm{chl} / \mathrm{mL}$ for $20 \mathrm{~min}$ at $4{ }^{\circ} \mathrm{C}$. The chloroplasts were centrifuged and resuspended in the isolation medium as described above.

Manganese Determination. The manganese content of the chloroplast membranes was generally measured by the atomic absorption method. Preparation of the samples was after Blankenship and Sauer (1974). One milliliter of the sample ( $3 \mathrm{mg}$ of $\mathrm{chl} / \mathrm{mL}$ ) was digested with $2 \mathrm{~mL}$ of $85 \%$ concentrated $\mathrm{HNO}_{3}$ and $15 \%$ concentrated $\mathrm{HClO}_{4}, \mathrm{v} / \mathrm{v}$, under gentle heating. The digested sample was filtered through Whatman no. 50 filter paper and brought to a final volume of $5 \mathrm{~mL}$ with distilled water. Measurements were made with a Perkin-Elmer Model 303 atomic absorption spectrometer. Standards at 0.2, $0.4,0.6,0.8$, and $1.0 \mathrm{ppm} \mathrm{Mn}$ were prepared in the buffer medium and carried through the same digestion procedure as the sample. Manganese determinations of the samples were interpolated from the standard curve which was linear and passed through the origin.

Alternatively, the manganese content was determined on sample aliquots by neutron activation analysis. The samples were heat sealed in precleaned polyethylene containers. Standards for comparison were prepared by pipetting $1 \mu \mathrm{L}$ of solutions of known manganese concentrations onto a Whatman no. 42 filter paper in precleaned vials. The samples and standards were then irradiated for $1 \mathrm{~h}$ at a flux of $2 \times 10^{12}$ neutrons/ $\mathrm{cm}^{2}-\mathrm{s}$ in the Illinois Advanced TRIGA Reactor facility. After a delay of 2 or $3 \mathrm{~h}$ to permit the decay of ${ }^{38} \mathrm{Cl}$, each sample was counted for 900 s using a $10 \%$ efficient Ge(Li) detector (Canberra) having a FWHM resolution of $2.1 \mathrm{keV}$ at $1333 \mathrm{keV}$. The $\gamma$-ray data were reduced to elemental concentrations using the program PIDAQ (developed by J. P. Maney, J. L. Fasching, and P. K. Hopke, University of Illinois). Two different $\gamma$-ray lines of ${ }^{56} \mathrm{Mn}$ were used to determine a weighted mean.

In both methods, several determinations were made of each preparation. The reproducibility was $\pm 1 \%$.

NMR Relaxation Measurements. Longitudinal $\left(T_{1}^{-1}\right)$ and transverse $\left(T_{2}^{-1}\right)$ relaxation rates were measured over a 4.7 to $70-\mathrm{MHz}$ range with a variable frequency pulsed spectrometer. The instrument employed a Varian (Model V-7400) 15-in. electromagnet with an operating range up to $24 \mathrm{kG}$. A Varian flux stabilizer (Model V-3508) and field-homogeneity unit (Model V-7530) provided adequate field stability and homogeneity. The output of the frequency synthesizer (Rockland Model 5600, 0.1 to $160 \mathrm{MHz}$ ) is split into two channels by the gating unit and is phase shifted for the pulse sequence of Carr and Purcell (1954) with the modification of Meiboom and Gill (1958). The A and B channels are gated by a pulse programmer through double-balanced mixers. The gated low-level $\mathrm{rf}$ is amplified using an Arenberg Model PG 650 tunable oscillator operated in the gated amplifier mode (this unit puts out up to $400 \mathrm{~W}$ ). The probe design (single coil, $6.5-\mathrm{mm}$ diameter) features interchangeable plug-in insert, vacuum Dewar assemblies. The $90^{\circ}$ pulse widths were $\sim 2 \mu \mathrm{s}$. The receiver is broadbanded $(3-150 \mathrm{MHz})$ and employs a Watkins-Johnson preamplifier (28-dB gain) and Kay Elemetrics amplifier $(40 \mathrm{~dB})$. A double-balanced mixer is the phase-sensitive detector, the output of which is applied to an operational amplifier for further gain and low-pass filtering.

The $T_{1}^{-1}$ measurements were made with the $180^{\circ}-\tau-90^{\circ}$ pulse sequence. Free induction decays (FID) following the $90^{\circ}$ pulse were collected on a storage oscilloscope for measurement of their amplitudes as a function of $\tau$. At least $8 \tau$ values were sampled and acquisitions were repeated at intervals of no less than $5 T_{1}$. The signal-to-noise ratio of the FID was sufficiently good even at the lowest frequency, so that semilog plots of the magnetization, In $\left[M_{0}-M(\tau)\right]$ vs. $\tau$, showed essentially no scatter and were linear over their whole range. The $T_{1}$ values are estimated to have an experimental uncertainty of about $\pm 5 \%$ and a deviation on a particular sample of not more than $\pm 3 \%$.

The $T_{2}^{-1}$ values were obtained from the decay of the echo envelope plotted on an oscillographic recorder. We used a delay of $2 \tau=1 \mathrm{~ms}$ between $180^{\circ}$ pulses. The $T_{2}^{-1}$ results have approximately the same error limits as $T_{1}{ }^{-1}$.

Probe temperature was controlled by a thermostatic flow of $\mathrm{N}_{2}$. A calibrated thermocouple encased in the NMR tube was used to measure the sample temperature before and after relaxation measurements. These readings varied by less than $1{ }^{\circ} \mathrm{C}$ after the temperature was stabilized.

Oxygen-17 Measurements. Chloroplast suspensions were prepared with water enriched to about $28 \%(w / w)$ in ${ }^{17} \mathrm{O}$. The water was purchased from the Mound Laboratory of the Monsanto Research Corp., Miamisburg, Ohio. The $T_{1}$ was measured by the inversion recovery method on the instrument 
described above. As a check, several measurements were repeated on a similar pulsed NMR spectrometer kindly made available to us by Dr. J. Jonas and his co-workers. Some continuous-wave (i.e., wide line) $T_{2}$ measurements were performed on a Varian WL-115 spectrometer. All measurements were made at room temperature. The pulsed NMR measurements were made at 8.1 and $12.8 \mathrm{MHz}$ and the wide-line measurements at $12.8 \mathrm{MHz}$.

Treatment of the Data. The proton relaxation rates observed as a function of frequency were fitted to a theoretical model whose details are outlined in the Theory section. A theoretical relaxation rate was calculated from the model, as a function of five adjustable parameters, for the frequency of each appropriately corrected experimental rate. Best-fit values were obtained for the five parameters by systematically varying them to minimize the differences between calculated and experimental rates. This least-squares type calculation was performed on an IBM 360/70 computer using the program MINROS, developed by Duane Steidinger, available in the computer program library at the University of Illinois.

\section{Theory}

The factors governing the proton relaxation rates (PRR) of aqueous systems containing low concentrations of paramagnetic species have been reviewed by Dwek (1973). In the dilute solutions of interest here, the paramagnetic contribution, i.e., the enhancement of the PRR, is given by

$$
(1 / T)_{\mathrm{p}}=\frac{p q}{T_{\mathrm{M}}+\tau_{\mathrm{M}}}+\frac{p q_{\mathrm{os}}}{T_{\mathrm{os}}}=(1 / T)_{\mathrm{obsd}}-(1 / T)_{\mathrm{d}}
$$

where $T$ stands for either $T_{1}$ or $T_{2}$, the $\left(T^{-1}\right)_{\text {obsd }}$ is the observed, exchange averaged PRR, $\left(T^{-1}\right)_{d}$ is the PRR for the system in the absence of the paramagnetic species, $p$ is the mole fraction of the paramagnetic species, $q$ is the hydration number of the paramagnetic species, $T_{M}$ is the relaxation time of protons in the hydration sphere of the paramagnetic site, $\tau_{M}$ is the mean lifetime of a proton in that sphere, and $T_{\mathrm{os}}$ is the relaxation time for the so-called "outer-sphere" protons, for which $q_{\text {os }}$ is the effective "hydration number."

The frequency dependences of $T_{\mathrm{M}}$ are given by the Solomon, Bloembergen, and Morgan (SBM) equations (Dwek, 1973) which are presented in a simplified form appropriate to the system studied here:

$$
\frac{1}{T_{1 \mathrm{M}}}=C\left[\frac{3 \tau_{\mathrm{c}}}{1+\omega_{1}^{2} \tau_{\mathrm{c}}{ }^{2}}+\frac{7 \tau_{\mathrm{c}}}{1+\omega_{\mathrm{S}}{ }^{2} \tau_{\mathrm{c}}^{2}}\right]
$$

and

$$
\frac{1}{T_{2 M}}=\frac{C}{2}\left[4 \tau_{\mathrm{c}}+\frac{3 \tau_{\mathrm{c}}}{1+\omega_{1}{ }^{2} \tau_{\mathrm{c}}{ }^{2}}+\frac{13 \tau_{\mathrm{c}}}{1+\omega_{\mathrm{s}}{ }^{2} \tau_{\mathrm{c}}{ }^{2}}\right]
$$

where $\omega_{1}$ and $\omega_{s}$ are the nuclear and electronic Larmor frequencies, respectively, and $C=(2 / 15) S(S+1) \gamma_{1}{ }^{2} \gamma_{S^{2}} r^{-6}$. In the latter, $S$ is the resultant electron spin-angular momentum, $\gamma_{1}$ and $\gamma_{S}$ are the nuclear and electronic magnetogyric ratios, respectively, and $r$ is the distance between a proton in the hydration sphere and the paramagnetic ion. In these equations, a term due to the electronic-nuclear scalar coupling has been omitted, since it has been shown to be negligible for $\mathrm{Mn}$ (II) bound to macromolecules (Navon, 1970).

The correlation time $\tau_{\mathrm{c}}$ is given as:

$$
\left(1 / \tau_{\mathrm{c}}\right)=\left(1 / \tau_{\mathrm{r}}\right)+\left(1 / \tau_{\mathrm{S}}\right)+\left(1 / \tau_{\mathrm{M}}\right)
$$

where $\tau_{\mathrm{r}}$ is the characteristic time for rotational reorientation of the paramagnetic complex and $\tau_{S}$ is the lifetime of an electronic spin state. The correlation times $\tau_{\mathrm{r}}$ and $\tau_{\mathrm{M}}$ are in- dependent of frequency. However, $\tau_{\mathrm{S}}$ is usually given by the following equation:

$$
\frac{1}{\tau_{\mathrm{S}}}=B\left[\frac{\tau_{\mathrm{v}}}{1+\omega_{\mathrm{S}}{ }^{2} \tau_{\mathrm{v}}^{2}}+\frac{4 \tau_{\mathrm{v}}}{1+4 \omega_{\mathrm{S}}{ }^{2} \tau_{\mathrm{v}}^{2}}\right]
$$

where $\tau_{\mathrm{v}}$ is the characteristic time for symmetry distortion of the complex and $B$ is a constant dependent upon the zero-field splitting of the paramagnetic complex. Therefore, $\tau_{\mathrm{S}}$ will be frequency dependent unless $\omega_{\mathrm{S}}^{2} \tau_{\mathrm{v}}^{2} \ll 1$.

The temperature dependence of $\tau_{M}$ is expected to show Arrhenius behavior, i.e.:

$$
\left(1 / \tau_{M}\right)=A \exp \left(-\Delta E^{\ddagger} / R T\right)
$$

The correlation times $\tau_{\mathrm{r}}$ and $\tau_{\mathrm{v}}$ are expected to obey a similar exponential relation, e.g., for $\tau_{\mathrm{r}}$ :

$$
\tau_{\mathrm{r}}=\tau_{\mathrm{r}}^{0} \exp \left(E_{\mathrm{a}} / R T\right)
$$

Outer sphere relaxation was found to contribute significantly to $T_{\mathrm{P}}^{-1}$ and could not be neglected in the treatment of the frequency-dependence data reported here. Two limiting cases (Dwek, 1973) have been suggested for the frequency dependences of $T_{o s}{ }^{-1}$ : (1) $T_{\mathrm{os}}{ }^{-1}$ are dominated by the correlation time $\tau_{S}$ and hence their frequency dependences are identical to those of $\tau_{\mathrm{M}}$, and (2) $T_{\mathrm{os}}{ }^{-1}$ are controlled by a correlation time which is related to the relative diffusional motion of the paramagnetic ion complex and the ligand protons. In the latter case, the values of $T_{\mathrm{os}}{ }^{-1}$ are given by the following equations:

$$
\left(1 / T_{10 \mathrm{~s}}\right)=D\left[7 f_{\mathrm{t}}\left(\omega_{\mathrm{s}} \tau_{\mathrm{s}}\right)+3 f_{\mathrm{t}}\left(\omega_{1} \tau_{\mathrm{s}}\right)\right]
$$

and

$$
\left(1 / T_{2 o s}\right)=D\left[\frac{13}{2} f_{\mathrm{t}}\left(\omega_{\mathrm{S}} \tau_{\mathrm{D}}\right)+3 f_{\mathrm{t}}\left(\omega_{1} \tau_{\mathrm{D}}\right)+2\right]
$$

where $\tau_{\mathrm{D}}$ is the characteristic time for diffusional motion of a proton, $f_{\mathrm{t}}$ is a function of $\omega$ and $\tau_{\mathrm{D}}$, and $D$ is a constant.

The above theory applies to dilute aqueous solutions of a single paramagnetic species. However, the chloroplast suspensions contain several paramagnetic species, so some thought need to be given to the separability of their effects on the PRR. Inspection of eq 1-3 shows that the effects of two paramagnetic centers (of the same or different species) will be additive if the presence of one does not affect the hydration $\left(q, q_{\mathrm{os}}, \tau_{\mathrm{M}}, r, \tau_{\mathrm{r}}\right)$ or spin-lifetime $\left(\tau_{S}\right)$ of the other. This is the case at infinite dilution and it is a good approximation in "dilute" solutions. However, at higher concentrations, direct or indirect interactions among the paramagnetic centers will lead to PRR that are nonlinear in the concentration(s) $p$ of the species involved.

Consider, for example, $\tau_{\mathrm{S}}$ which is probably the parameter most likely to be affected significantly by other paramagnetic centers. Besides the zero-field splitting term given in eq 5 , there is diffusional, dipole-dipole relaxation among the centers, which adds a concentration-dependent term, that is:

$$
\left(1 / \tau_{\mathrm{S}}\right)=\left(1 / \tau_{\mathrm{S}}\right)_{\mathrm{v}}+A p
$$

where $A$ is a phenomenologically determined constant. Substitution of this expression in eq 1-4 shows that for high concentrations of paramagnetic species $(1 / T)_{\mathrm{p}}$ becomes proportional to $p^{2}$. There can be a similar, rotational, dipole-dipole contribution to $\tau_{\mathrm{S}}$ if two paramagnetic centers are held close to one another by tight binding to the same macromolecule.

Results

Dependence of the PRR upon Mn Content. Chloroplast membranes contain two pools of manganese (Cheniae, 1970), 


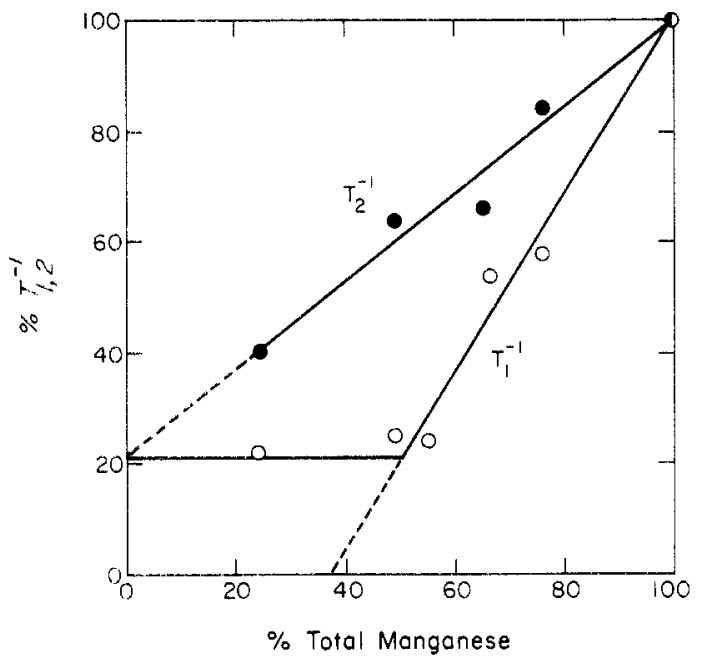

FIGURE 1: Proton relaxation rates as a function of manganese content, plotted as percent of the values for an unwashed control containing 0.62 $\pm 0.03 \mu \mathrm{g}$ of $\mathrm{Mn} / \mathrm{mg}$ of chl. The PRR were measured at $27 \mathrm{MHz}$ and 25 ${ }^{\circ} \mathrm{C}$ on dark-adapted pea chloroplast suspensions containing $3 \mathrm{mg}$ of $\mathrm{chl} / \mathrm{mL}$, and corrected for buffer contributions. The manganese content was varied by $\mathrm{Mg}$ exchange.

a "tightly" bound and a "loosely" bound fraction. The latter, which represents approximately two-thirds of the total manganese, has been associated with oxygen evolution. Several treatments cause the release of the loosely bound manganese from the membrane and the concurrent appearance of a Mn(II) EPR signal (Lozier et al., 1971); these include incubation with alkaline Tris buffer (Yamashita and Butler, 1968), hydroxylamine (Cheniae and Martin, 1971), or high concentrations of $\mathrm{MgCl}_{2}$ (Chen and Wang, 1974). In our experiments the chloroplasts were incubated at different $[\mathrm{Mg}] /[\mathrm{chl}]$ ratios, thereby removing an adjustable fraction of the initial manganese.

Figure 1 shows the relaxation rates that result from this treatment. The normalized enhancement of the proton relaxation in the treated suspensions is plotted as a function of the residual fraction of manganese, in percent. The normalized enhancement was obtained by subtracting the relaxation rate of the buffer from the relaxation rate observed for the treated suspension, per eq 1, and dividing by the enhancement of the untreated suspension. If all of the enhancement were due to manganese which is homogeneous in its effects upon the relaxation rates, the data would fall upon a single straight line extending from the origin to the upper right hand corner of the plot. Instead, the $T_{2}^{-1}$ data in Figure 1 lie above such a line, while the $T_{1}^{-1}$ data are below.

A simple interpretation of these results is the following: (1) About $20 \%$ of the enhancement in the untreated suspensions is caused by species other than manganese in the chloroplasts. (2) The tightly and loosely bound fractions of manganese are of comparable effect, per atom, upon $T_{2}^{-1}$. (3) The tightly bound fraction of manganese has little effect upon $T_{1}^{-1}$. Point 1 is based upon extrapolation of the $T_{1}{ }^{-1}$ and $T_{2}^{-1}$ data to zero manganese, both of which give an intercept of $20 \%$ enhancement. Point 2 is indicated by the linearity of the $T_{2}^{-1}$ plot, including concentrations at which some tightly bound $\mathrm{Mn}$ has been removed. Point 3 is based upon the two straight-line portions in the $T_{1}^{-1}$ plot.

Figure 2 shows the values of $T_{1}^{-1}$ observed for dark-adapted chloroplast suspensions as a function of NMR frequency. Part a of the figure shows the original $T_{1}^{-1}$ data, which are affected by small differences in $\mathrm{Mn}$ concentration. The manganese

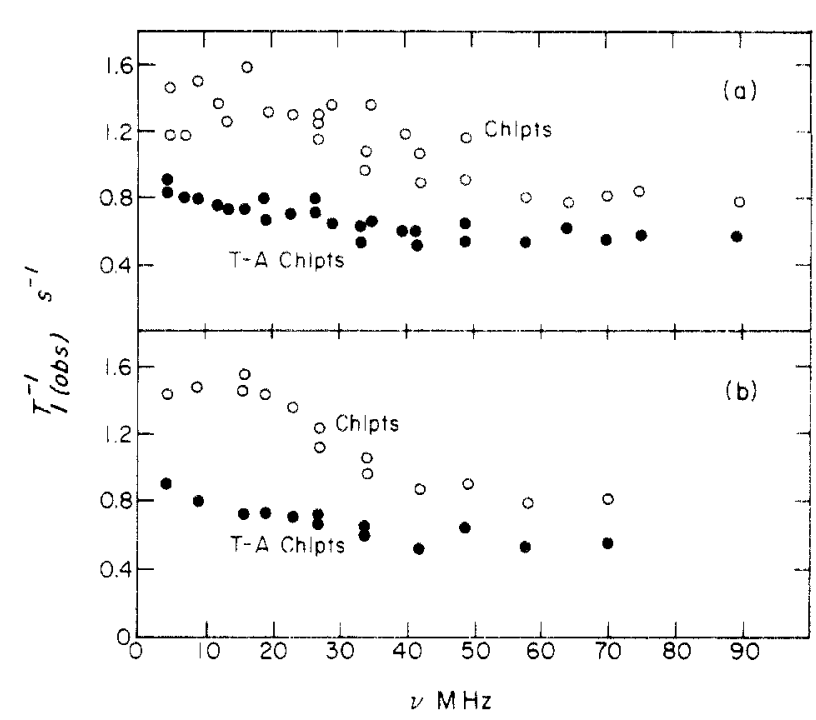

FIGURE 2: Frequency dependence of the proton $T_{1}^{-1}$ observed for dark-adapted pea chloroplast suspensions (chlpts) containing $3 \mathrm{mg}$ of $\mathrm{chl} / \mathrm{mL}$, at room temperature $\left(23-25^{\circ} \mathrm{C}\right)$. (a) Uncorrected rates observed for several different samples. (b) Observed rates adjusted to the same $\mathrm{Mn}$ concentration. T-A chlpts refers to Tris-acetone washed chloroplasts in which the loosely bound fraction of $\mathrm{Mn}$ is removed.

content was determined for seven of the chloroplast preparations, five by atomic absorption and two by neutron activation. The average values for these preparations ranged from 0.400 to $0.496 \mu \mathrm{g}$ of $\mathrm{Mn} / \mathrm{mg}$ of chl, a variation comparable with that reported by Blankenship and Sauer (1974). In part b of Figure 2 , the $T_{1}^{-1}$ data for these seven preparations have been multiplied by the appropriate factors to adjust them to a single $\mathrm{Mn}$ concentration, $2.7 \times 10^{-5} \mathrm{M}(0.5 \mu \mathrm{g}$ of $\mathrm{Mn} / \mathrm{mg}$ of chl). (Some of the data in part a are from early preparations for which the Mn content was not determined.) It is clear that most of the scatter of the data in part a is due to variations in the $\mathrm{Mn}$ content of the different preparations.

This observation and the data in Figure 1 demonstrate that the major part of the enhancement in the PRR is caused by the loosely bound $\mathrm{Mn}$. Our next step is to consider separation of the proton relaxation by the loosely bound $\mathrm{Mn}$ from that of the other paramagnetic species in the chloroplasts. If this is feasible, information about the nature of the complex containing the loosely bound Mn can be sought from the frequency and the temperature dependences of its effects.

There is substantial evidence that the different paramagnetic species give additive contributions to the PRR. For example, it has been pointed out (Bloembergen and Morgan, 1961) that interactions among $\mathrm{Mn}^{2+}$ ions play a negligible role in the proton relaxation of aqueous solutions at concentrations below $0.05 \mathrm{~N}$, which is a hundred times more concentrated than $\mathrm{Mn}$ in the chloroplast suspensions. In Figure 1, removal of $\mathrm{Mn}$ from the chloroplasts reduces their enhancement of the PRR in linear proportion. Also, the proton $T_{1}^{-1}$ was found to be linear in the amount of chlorophyll over a tenfold concentration range, from 0.5 to $5.0 \mathrm{mg}$ of $\mathrm{chl} / \mathrm{mL}$, at $27 \mathrm{MHz}$ and $25^{\circ} \mathrm{C}$, using pea chloroplasts. Such linear behavior would be expected for additive contributions in a homogeneous system, as pointed out in the discussion of eq 10 . However, the chloroplast suspensions are heterogeneous and the observed linearities do not eliminate a rotational contribution to $\tau_{\mathrm{S}}$ by interactions between paramagnetic centers selectively bound to neighboring sites on a macromolecule. But any such term (analogous to eq 5 ) is unlikely to be significant because the tumbling time $\tau_{r}$ for $\mathrm{Mn}$ bound to a membrane is expected to be relatively long 


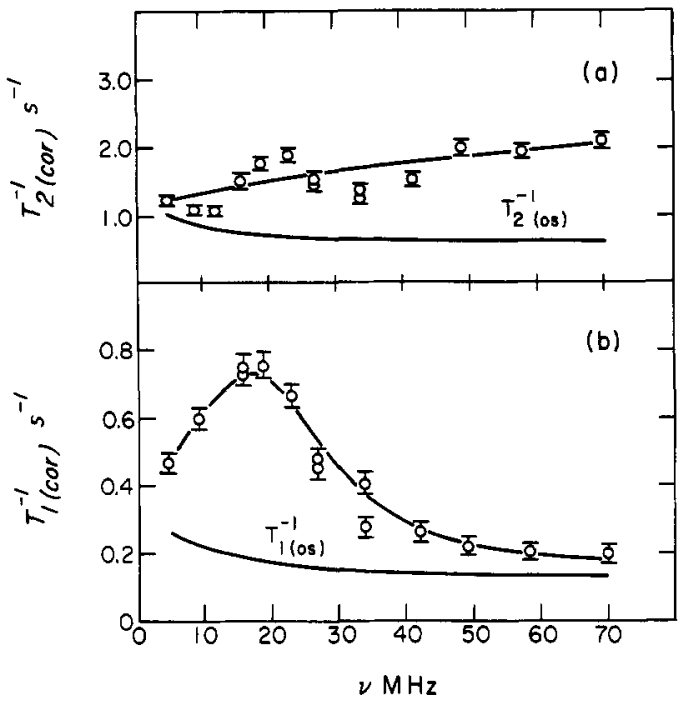

FIGURE 3: Frequency dependence of the PRR for the loosely bound paramagnetic species in dark-adapted suspensions of pea chloroplasts $(3$ $\mathrm{mg}$ of chl $/ \mathrm{mL}$ ). The open circles are experimental values measured at room temperature $\left(23-25^{\circ} \mathrm{C}\right)$, corrected for the tightly bound species and adjusted to the same Mn concentration. The upper, solid lines are theoretical best fits of the data corresponding to the parameters in Table II. The lower solid lines are theoretical outer-sphere contributions based on a diffusional model.

(Dwek, 1973). This leads to $\omega_{\mathrm{S}}^{2} \tau_{\mathrm{r}}^{2} \gg 1$ and a negligible contribution to $\tau$ s.

Nature of the Loosely Bound Mn. The evidence just cited makes it reasonable to attempt to separate the effects of the loosely bound $\mathrm{Mn}$ from those of the other paramagnetic species. The open circles in both parts of Figure 2 are $T_{1}^{-1}$ for untreated chloroplasts. The $T_{1}^{-1}$ data labeled $\mathrm{T}-\mathrm{A}$ chlpts (Tris-acetone washed chloroplasts) are for chloroplasts which have had the loosely bound fraction of $\mathrm{Mn}$ removed by washing with Tris buffer containing $20 \%$ acetone. The difference between the two rates should be a good approximation to the enhanced relaxation caused by the loosely bound Mn. Such "corrected" relaxation rates $\left(T_{2}^{-1}\right.$ as well as $\left.T_{1}{ }^{-1}\right)$ have been obtained by subtracting the two sets of rates, adjusted to the same Mn concentration, i.e.,

$$
(1 / T)_{\text {cor }}=(1 / T)_{\text {obsd }}-(1 / T)_{\mathrm{T}-\mathrm{A} \text { chlpts }}
$$

The results for $(1 / T)_{\text {cor }}$ are given in Figure 3. In general appearance they are quite similar to those for $\mathrm{Mn}$ (II) complexes, i.e., a severalfold value for $T_{2} / T_{1}$ and a broad maximum in $T_{1}^{-1}$ at lower frequencies $(\nu \approx 20 \mathrm{MHz})$. In contrast, for the residual paramagnetic species, e.g., $\left(1 / T_{1}\right)_{T-A}$ chlpts in Figure 2, we find $T_{2} / T_{1} \approx 2$; also, both $T_{2}{ }^{-1}$ (not shown) and $T_{1}^{-1}$ are virtually independent of frequency except for a small increase in $T_{1}^{-1}$ at lower frequencies. This behavior is characteristic of paramagnetic ions with a short. $\tau_{\mathrm{S}}\left(\sim 10^{-10} \mathrm{~s}\right)$ such as Fe(III) (Villafranca et al., 1974).

A quantitative treatment of the data in Figure 3 can be accomplished with the simplified SBM equations for the case in which $\tau_{\mathrm{S}}$ is frequency dependent and makes a sizable contribution to $\tau_{\mathrm{c}}{ }^{-1}$. Attempts to fit the data assuming only $\tau_{\mathrm{S}}$ relaxation (i.e., $\tau_{c}^{-1}=\tau_{\mathrm{S}}{ }^{-1}$ ) failed and it was necessary to include an additional frequency independent correlation time in the analysis (i.e., either $\tau_{\mathrm{M}}, \tau_{\mathrm{r}}$, or both). However, as pointed out at the end of the preceding section, $\tau_{\mathrm{r}}$ is probably relatively long and will have little effect on $\tau_{c}$; therefore, it-was assumed that the frequency-independent term in $\tau_{c}{ }^{-1}$ arises from $\tau_{M}$ so that $\tau_{\mathrm{c}}{ }^{-1}=\tau_{\mathrm{S}}{ }^{-1}+\tau_{\mathrm{M}}{ }^{-1}$. While this approach fits the qualitative features of the $T_{1}^{-1}$ and $T_{2}^{-2}$ dependences upon
TABLE 1: Relaxation Parameters Found for Protons in Aqueous Solutions of Chloroplasts and in Several Known Mn(II) Systems.

\begin{tabular}{|c|c|c|c|}
\hline & $\begin{array}{c}\tau \mathrm{s}^{0} \\
\left(10^{-8} \mathrm{~s}\right) \\
\end{array}$ & $\begin{array}{c}\begin{array}{c}\tau_{\mathrm{M}} \\
\left(10^{-8} \mathrm{~s}\right)\end{array} \\
\end{array}$ & $\begin{array}{c}B \\
\left(10^{19} \mathrm{rd}^{2} \mathrm{~s}^{-2}\right) \\
\end{array}$ \\
\hline Chlpts & $1.1 \pm 0.1$ & $2.2 \pm 0.2$ & $0.90 \pm 0.09$ \\
\hline Chlpts $+\mathrm{Ph}_{4} \mathrm{~B}$ & $1.1 \pm 0.1$ & $1.0 \pm 0.1$ & $0.90 \pm 0.09$ \\
\hline Chlpts + FeCy & $2.0 \pm 0.2$ & $0.1 \pm 0.01$ & $1.1 \pm 0.1$ \\
\hline Aqueous $\mathrm{Mn}(\mathrm{II})^{a}$ & $0.95 \pm 0.1$ & 2.3 & 10 \\
\hline $\begin{array}{c}\mathrm{Mn}(\mathrm{II}) \text {-carboxy- } \\
\text { peptidase } \mathrm{A}^{b}\end{array}$ & $0.92 \pm 0.1$ & $0.25 \pm 0.3$ & $3.1 \pm 0.4$ \\
\hline $\begin{array}{l}\text { Mn(II)-pyruvate } \\
\text { kinase }^{b, c}\end{array}$ & $\begin{array}{l}2.3 \pm 0.6 \\
2.3\end{array}$ & $\begin{array}{l}0.4 \pm 0.04 \\
0.5 \pm 0.1\end{array}$ & $\begin{array}{l}0.8 \pm 0.1 \\
1.46\end{array}$ \\
\hline $\begin{array}{l}{ }^{a} \text { Bloemberger } \\
\text { nd Cohn }(1970\end{array}$ & Iaran 1 & & 70). \\
\hline
\end{tabular}

frequency, it does not account for the large $T_{1}^{-1}$ at high frequencies (i.e., $\nu \geq 50 \mathrm{MHz}$ ) or the large values of $T_{2}^{-1}$ at low frequencies $(\nu \leq 30 \mathrm{MHz})$.

The discrepancies cannot be removed by adjusting the scale of the "background" correction, $(1 / T)_{\mathrm{T}-\mathrm{A} \text { chlpts }}$ in eq 11. Instead, the data were successfully fitted by including the terms $T_{\text {os }}$ of eq 1 in the analysis and assuming that the diffusional model correctly describes their frequency dependence. The solid lines passing through the data points in Figure 3 have been calculated on this basis from eq $1,9,10$, and the SBM equations. The calculated curves marked $T_{10 \mathrm{~s}}^{-1}$ and $T_{2 \mathrm{os}^{-1}}$ show the contributions of these terms. However, the source of this contribution is uncertain, and the treatment presented here, although reasonable, is empirical. For this reason, no physical significance is attached to the parameters $D$ and $\tau_{D}$ of eq 8 and 9 , obtained in the fitting process. Similarly, no attempt was made to interpret the best-fit value of $C$ (eq 3 and 4 ).

Table I lists the values of $\tau S^{0}, \tau_{M}$, and $B$ for dark-adapted chloroplasts, obtained from the fitting process for the data of Figure 3. The superscript 0 designates the value of $\tau_{\mathrm{S}}$ at zero field, i.e., $\omega_{S}=0$ in eq 5 . Also listed in Table I are these parameters evaluated by other workers from data on aqueous systems containing known macromolecular $\mathrm{Mn}$ (II) complexes. There are close similarities among the several sets of values. On the other hand, $\mathrm{Mn}$ (III) is quite different. In particular, $\tau_{\mathrm{S}}^{0} \approx 10^{-8} \mathrm{~s}$ for $\mathrm{Mn}(\mathrm{II})$ (Dwek, 1973), while it has been estimated to be $\approx 10^{-11}$ s for Mn(III) (Villafranca et al., 1974). The latter value is too small to account adequately for the observed relaxation data; e.g., it does not predict the maximum in $\left(1 / T_{1}\right)_{\text {cor }}$ at $\sim 17 \mathrm{MHz}$. Therefore, we conclude that $\mathrm{Mn}(\mathrm{II})$ is the species of manganese governing the PRR in the chloroplast suspensions.

With the values given above for the various parameters, $T_{M}$ and $\tau_{M}$ were calculated and it was found that $\tau_{M} \ll T_{M}$ at all frequencies employed. This result is confirmed by the temperature dependence of the PRR shown in Figure 4. The data exhibit the non-Arrhenius type of behavior that occurs when more than one rate process contributes significantly to the relaxation, $\tau_{\mathrm{S}}$ (or $\tau_{\mathrm{v}}$ ) and $\tau_{\mathrm{M}}$ in this case. The changing slope of the curved lines gives an estimated activation energy of 2-3 $\mathrm{kcal} / \mathrm{mol}$. This range is intermediate to the values expected for electronic relaxation via $\tau_{\mathrm{v}}(\sim 1 \mathrm{kcal} / \mathrm{mol})$ and for chemical exchange $(5-15 \mathrm{kcal} / \mathrm{mol})$. The limited temperature range available to the system $\left(5-38^{\circ} \mathrm{C}\right)$ prevents one from obtaining accurate activation energies for $\tau_{\mathrm{S}}$ and $\tau_{\mathrm{M}}$ separately.

Effects of Redox Agents. The addition of redox agents to the chloroplast suspensions produces changes that are in accord with loosely bound $\mathrm{Mn}$ (II) being the chief source of the proton relaxation. The higher oxidation states of manganese are not 


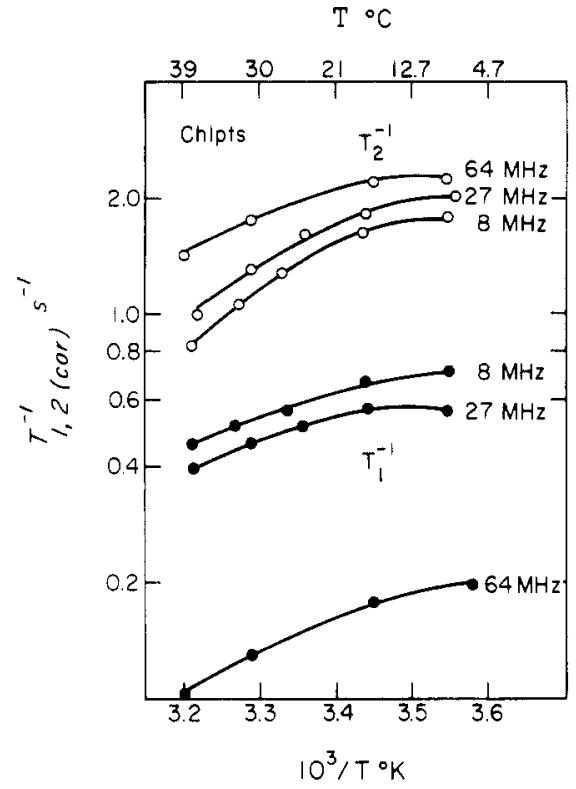

FIGURE 4: A semi-log plot of the temperature dependence of the PRR for the loosely bound paramagnetic species in dark-adapted suspensions of pea chloroplasts ( $3 \mathrm{mg}$ of $\mathrm{chl} / \mathrm{mL}$ ) at several frequencies. The experimental values have been corrected for the tightly bound species and adjusted to the same Mn concentration.

readily reduced beyond $\mathrm{Mn}$ (II), but the latter is oxidized quite easily. Thus, if the Mn were present in dark-adapted chloroplasts as a mixture of $\mathrm{Mn}(\mathrm{II})$ and some higher oxidation state(s), e.g., $\mathrm{Mn}$ (III), the addition of a reducing agent could convert the $\mathrm{Mn}(\mathrm{III})$ to $\mathrm{Mn}(\mathrm{II})$, thereby increasing the PRR. On the other hand, an oxidizing agent could convert the Mn(II) to $\mathrm{Mn}(\mathrm{III})$, decreasing the PRR.

Earlier work (Wydrzynski et al., 1975) has shown that reductants do increase the PRR of untreated chloroplasts, while oxidants cause decreases. However, if the loosely bound Mn is removed by washing with Tris-acetone, redox agents have little effect. In the present work, the $T_{1}^{-1}$ for a washed chloroplast suspension was found to be $0.21 \mathrm{~s}^{-1}$ compared with 0.82 $\mathrm{s}^{-1}$ for an unwashed control. The presence of the reductant tetraphenylboron $\left(\mathrm{Ph}_{4} \mathrm{~B}\right)$ at $5 \mathrm{mM}$ in the washed sample gave $0.28 \mathrm{~s}^{-1}$ and the oxidant potassium ferricyanide ( $\mathrm{FeCy}$ ) at 50 $\mathrm{mM}$ gave $0.23 \mathrm{~s}^{-1}$. Furthermore, the frequency dependences of $T_{1}{ }^{-1}$ and $T_{2}{ }^{-1}$ were found to be the same for T-A washed chloroplasts with and without $\mathrm{FeCy}$.

Frequency-dependence studies were made of the PRR for dark-adapted suspensions of unwashed pea chloroplasts, in one case with $\mathrm{PH}_{4} \mathrm{~B}$ at $5 \mathrm{mM}$ and in the other with $\mathrm{FeCy}$ at 50 $\mathrm{mM}$. The results are given in Figure 5 . The behavior in the presence of $\mathrm{Ph}_{4} \mathrm{~B}$ is very similar to that for the control (the suspensions without $\mathrm{Ph}_{4} \mathrm{~B}$ in Figure 3), except that the rates are three to four times greater. This is also the case for the temperature dependences of $(1 / T)_{\text {cor }}$ at 8,24 , and $64 \mathrm{MHz}$ for chloroplasts with $\mathrm{Ph}_{4} \mathrm{~B}$ added. In contrast, the rates in the presence of $\mathrm{FeCy}$ are somewhat less than those of the control, and the frequency dependences differ. Analysis of the data gives the best fit parameters included in Table I for the Mn(II) contribution.

Separation of the outer-sphere terms in the fitting process was only semiquantitative because they are not much larger than experimental error. But it is clear that both the $T_{1}^{-1}$ and $T_{2}^{-1}$ contributions are increased by $\mathrm{Ph}_{4} \mathrm{~B}$, the $T_{1}^{-1}$ severalfold and $T_{2}^{-1}$ less than twofold. Addition of $\mathrm{FeCy}$, on the other hand, causes $T_{2}{ }^{-1}$ to decrease but seems to have little effect upon $T_{1}^{-1}$. It seems likely that much of what we lump together

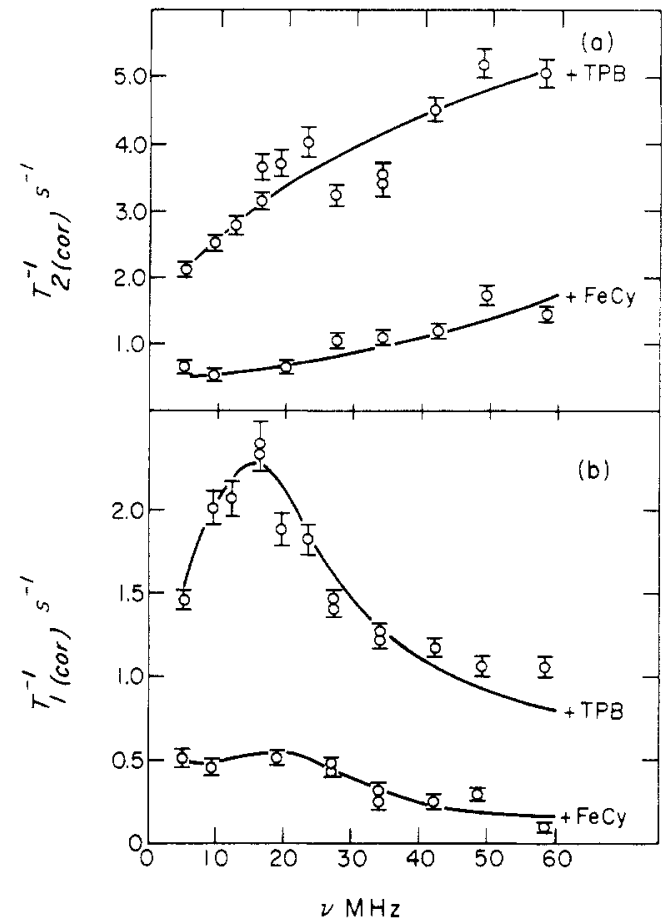

FIGLRE 5: Frequency dependence of the PRR for the loosely bound paramagnetic species in dark-adapted suspensions of pea chloroplasts ( 3 $\mathrm{mg}$ of $\mathrm{chl} / \mathrm{mL}$ ) in the presence of redox agents, with conditions otherwise as in Figure 3. The $\mathrm{Ph}_{4} \mathrm{~B}$ (TPB) was $5 \mathrm{mM}$ and the $\mathrm{FeCy}$ was $50 \mathrm{mM}$. The parameters from the best-fit, theoretical solid lines are given in Table II.

in the outer-sphere terms is caused by Mn(II). However, the terms are still relatively large for suspensions in which most of the enhancement characteristic of $\mathrm{Mn}$ (II) has been removed by $\mathrm{FeCy}$. Therefore, the terms for the untreated chloroplasts probably include appreciable contributions from paramagnetic species other than $\mathrm{Mn}$ (II) that are removed by the Tris-ace. tone washing. Furthermore, Mn(III) is an unlikely candidate for this role because of its small molar relaxivity (Villafranca et al., 1974).

These experiments with redox agents confirm that the PRR are determined primarily by the amount of $\mathrm{Mn}(\mathrm{II})$ in the chloroplast suspensions. In addition, the results show that the loosely bound $\mathrm{Mn}$ in untreated chloroplasts is a mixture of $\mathrm{Mn}(\mathrm{II})$ and a higher oxidation state(s), perhaps $\mathrm{Mn}$ (III). About one-fourth of the loosely bound $\mathrm{Mn}$ occurs as $\mathrm{Mn}$ (II) in the dark-adapted, untreated chloroplasts. There is some evidence for several types of $\mathrm{Mn}$ sites and/or states. A titration curve for spinach chloroplasts ( $3 \mathrm{mg}$ of chl $/ \mathrm{mL}$ ) of $T_{1}{ }^{-1} \mathrm{vs}$. $\mathrm{Ph}_{4} \mathrm{~B}$, at $27 \mathrm{MHz}$ and $25^{\circ} \mathrm{C}$, shows two distinct plateaus, one at 1-2 $\mathrm{mM}$ and the other above $4 \mathrm{mM}$, with perhaps a third plateau above $15 \mathrm{mM}$ (Wydrzynski et al., 1976b).

Exchange of Water across Thylakoid Membranes. Oxygen-17 NMR data can complement the PRR because relaxation of the oxygen-17 is governed by exchanges of whole water molecules between the solvent and solvation sphere of a paramagnetic center, while proton exchange dominates the PRR. One would expect different exchange rates for the two species in at least some circumstances, e.g., across membranes. Results obtained for oxygen-17 that bear on this point are summarized in Table II. In no case was there any evidence of other than a single relaxation time. The $T^{-1}$ values observed for the various samples have been corrected by subtracting from them the relaxation rates found for buffers containing the same species except the chloroplasts. 
TABLE II: Results of Oxygen-17 NMR Relaxation Rate Measurements for Chloroplast Suspensions. ${ }^{a, b}$

\begin{tabular}{|c|c|c|c|c|c|}
\hline \multirow[b]{2}{*}{ Sample } & \multicolumn{3}{|c|}{ rf pulse expts } & \multicolumn{2}{|c|}{ Wide line expts ${ }^{c}$} \\
\hline & $\begin{array}{c}\left(T_{1}^{-1}\right)_{\text {obsd }} \\
\left(\mathrm{s}^{-1}\right)\end{array}$ & $\begin{array}{c}\left(T_{1}^{-1}\right)_{\mathrm{cor}} \\
\left(\mathrm{s}^{-1}\right)\end{array}$ & $\begin{array}{c}\text { Freq } \\
(\mathrm{MHz})\end{array}$ & $\begin{array}{c}\left(T_{2}^{-1}\right)_{\text {obsd }} \\
\left(\mathrm{s}^{-1}\right)\end{array}$ & $\begin{array}{c}\left(T_{2}^{-1}\right)_{\text {cor }} \\
\left(\mathrm{s}^{-1}\right)\end{array}$ \\
\hline $\begin{array}{l}\text { Control } \\
\mathrm{Ph}_{4} \mathrm{~B}(5 \mathrm{mM}) \\
\mathrm{T}-\mathrm{A} \text { washed }\end{array}$ & $\begin{array}{l}199^{d} \\
192 \\
198\end{array}$ & $\begin{array}{r}11 \\
4 \\
10\end{array}$ & $\begin{array}{l}12.8 \\
12.8 \\
12.8\end{array}$ & & \\
\hline $\begin{array}{l}\text { Control } \\
\text { Triton- } \mathrm{X}^{\mathrm{R}}\end{array}$ & $\begin{array}{l}182^{d} \\
246\end{array}$ & $\begin{array}{l}14 \\
37^{e}\end{array}$ & $\begin{array}{l}8.1 \\
8.1\end{array}$ & $\begin{array}{l}613^{d} \\
683\end{array}$ & $\begin{array}{c}0 \\
157^{e}\end{array}$ \\
\hline
\end{tabular}

${ }^{a}$ All measurements were with the sample at $25 \pm 1{ }^{\circ} \mathrm{C} .{ }^{b}$ The reproducibility of the values given is $\pm 5 \%{ }^{c}$ The wide line experiments were at a frequency of $12.8 \mathrm{MHz} .{ }^{d}$ The control sample contained $2 \mathrm{mg}$ of $\mathrm{Chl} / \mathrm{mL}$ of untreated chloroplasts plus the usual buffer. ${ }^{e}$ In determining these values, the same amount of detergent $(\mathrm{v} / \mathrm{v})$ was added to the buffer and to the suspension.

It is seen that, within experimental error, the addition of redox agents has no effect upon the oxygen-17 $T_{1}^{-1}$ of the chloroplast suspensions. This indicates that the water molecules in the solvent phase exchange too slowly with the small fraction of water molecules in the solvation sphere of the loosely bound $\mathrm{Mn}(\mathrm{II})$ to be affected by them. The limiting rate might, in principle, be either the chemical exchange itself or slow exchange of water across the membranes to $\mathrm{Mn}$ (II) located inside the thylakoids. In order to distinguish between these two possibilities, the chloroplasts were treated with the detergent Triton- $X^{R}(1 \%, v / v)$ which solubilizes the thylakoid membranes and exposes their interior to the bulk water. This treatment caused the oxygen-17 $T_{1}{ }^{-1}$ and $T_{2}{ }^{-1}$ to increase appreciably, as shown by the data in Table II.

A similar set of experiments was performed on solutions of $6 \times 10^{-5} \mathrm{M} \mathrm{Mn}^{2+}$ in the buffer, with and without the detergent. The oxygen-17 $T_{2}^{-1}$ from the line width $\left(500 \mathrm{~s}^{-1}\right.$ in the buffer alone) was found to be only slightly broader $\left(960 \mathrm{~s}^{-1}\right)$ with the detergent than without $\left(900 \mathrm{~s}^{-1}\right)$, well within experimental error. This indicates that the detergent has little or no direct effect upon $\tau_{M}$ or the relaxation rates for oxygen-17 in $\mathrm{Mn}$ (II) complexes. We conclude therefore that the detergent increases the relaxation rates of the chloroplast suspensions by exposing the bulk water population to the $\mathrm{Mn}$ (II) originally inside the thylakoids.

\section{Discussion}

There are several paramagnetic species besides $\mathrm{Mn}(\mathrm{II})$ in chloroplast membranes which may contribute to the PRR. However, we expect the effects of these ions to be small at the concentrations present in the samples used in this study. $\mathrm{Mn}$ (II) is probably the most efficient proton relaxer of all ions in the first-row transition elements (Mildvan and Cohn, 1970) due to its relatively long electronic correlation time. High-spin $\mathrm{Fe}(\mathrm{II}), \mathrm{Fe}(\mathrm{III})$ (Dwek, 1973), and Mn(III) (Villafranca et al., 1974 ) have short electronic relaxation times $\left(\tau_{\mathrm{S}} \approx 10^{-10} \mathrm{~s}\right)$ and the effects of these ions are comparatively small even though they may be present at higher concentrations than the $\mathrm{Mn}$ (II). On the other hand, the electronic relaxation time of $\mathrm{Cu}$ (II) is comparable to $\mathrm{Mn}$ (II), but in chloroplasts at least half of the copper is located in plastocyanin which itself has no effect on the PRR, since the bound copper is not accessible to the solvent water (Blumberg and Peisach, 1966). The remaining copper in chloroplasts is mostly located in polyphenyloxidase, an enzyme not associated with photosynthesis and perhaps is washed out during the chloroplast preparation method used here. The contributions of these ions could nonetheless account for the $20 \%$ background in the PRR found in Figure 1.

Also, the results in Figure 1 indicate that $T_{1}^{-1}$ is sensitive primarily to the loosely bound fraction of $\mathrm{Mn}$ (about two-thirds of the total Mn content of the membrane), while the $T_{2}{ }^{-1}$ appears to reflect the total $\mathrm{Mn}$ content. This difference in proton relaxation by loosely and tightly bound $\mathrm{Mn}$ is not too surprising. In fact, the insensitivity of $T_{1}{ }^{-1}$ to frequency for the tightly bound $\mathrm{Mn}$ (the T-A chloroplast curve in Figure $2 \mathrm{~b}$ ) shows that, whatever its oxidation state, its $\tau_{\mathrm{S}}$ differs substantially from that for the loosely bound Mn. Moreover, differences would be expected in $\tau_{\mathrm{M}}$, the time required for protons to exchange from the solvation sphere to the $\mathrm{Mn}$. In turn, such differences in $\tau_{S}$ and $\tau_{M}$ could cause the tightly bound $\mathrm{Mn}$ to have a small effect on $T_{1}{ }^{-1}$ but a large effect on $T_{2}{ }^{-1}$.

The exchange of whole water molecules across biological membranes has been calculated to be relatively slow. For example, the average lifetime of a water molecule inside human erythrocytes has been given as $17 \mathrm{~ms}$ (Vieira et al., 1970; Shporer and Civan, 1975; Andrasko, 1976). It has been noted that such slow exchange could limit nuclear relaxation by paramagnetic species inside the membranes (Case, 1975). The suspensions of chloroplast vesicles contain three water fractions: internal and external fractions plus the solvation sphere of the Mn(II). Nuclei in the solvation sphere of the Mn(II) have greatly enhanced relaxation rates (eq 1 with $p q=1$ ). Sharing of the enhancement with the other solvent nuclei depends upon the exchange rates among the three fractions and upon their relative populations (Gutowsky et al., 1965). Current models of chloroplast thylakoids place the manganese associated with oxygen evolution on the inside of the chloroplast vesicles (Trebst, 1974). The results of the preceding section confirm this location and show that exchange of water $\left({ }^{17} \mathrm{O}\right)$ across the membrane is too slow to average the different relaxation rates of ${ }^{17} \mathrm{O}$ in the external and internal water. However, inside the vesicles, chemical exchange does average the bulk water with that in the solvation sphere of the $\mathrm{Mn}(\mathrm{II})$.

These observations may be used to place a lower limit upon the average lifetime $\tau_{\mathrm{i}}$ of water molecules inside the vesicles. The exchange across the membranes will not average out the difference in the relaxation rates provided that (Gutowsky et al., 1965) the average rate constant for exchange is less than the difference between external and internal relaxation rates; that is

$$
k_{\mathrm{av}} \equiv 0.5\left[\left(1 / \tau_{\mathrm{e}}\right)+\left(1 / \tau_{\mathrm{i}}\right)\right]<\left|T_{\mathrm{e}}^{-1}-T_{\mathrm{i}}^{-1}\right|
$$

where $k=1 / \tau$ is a rate constant for exchange across the membrane, the subscripts $e$ and $i$ refer to nuclei external to and inside of the vesicles, and the subscript av designates an average over the two fractions. The exchange rate across the membrane is the same in both directions,

$$
p_{\mathrm{c}} / \tau_{\mathrm{e}}=p_{\mathrm{i}} / \tau_{\mathrm{i}}
$$


Furthermore, even though the suspensions contained high chlorophyll concentrations ( $2 \mathrm{mg}$ of $\mathrm{chl} / \mathrm{mL}$ ), the volume of internal water is only about $3-4 \%$ of the total, giving $p_{\mathrm{e}} \approx 1$ and $1 / \tau_{\mathrm{i}} \gg 1 / \tau_{\mathrm{e}}$. Thus, we have

$$
k_{\mathrm{av}} \approx 0.5 / \tau_{\mathrm{i}}
$$

The right-hand side of eq 12 can be evaluated by noting that the relaxation rates $T_{\mathrm{b}}{ }^{-1}$ for the buffer without chloroplasts are $T_{\mathrm{e}}^{-1}$, while $T_{\mathrm{i}}^{-1}$ is determinable from the exchange-averaged relaxation rates $T_{\mathrm{d}}^{-1}$ for the detergent-treated chloroplasts by the relation

$$
T_{\mathrm{d}}^{-1}=T_{\mathrm{av}}^{-1}=p_{\mathrm{e}} T_{\mathrm{e}}^{-1}+p_{\mathrm{i}} T_{\mathrm{i}}^{-1} \approx T_{\mathrm{b}}^{-1}+p_{\mathrm{i}} T_{\mathrm{i}}^{-1}
$$

By combining eq 12-15 and introducting the definition $T_{\text {cor }}{ }^{-1}$ $=T_{\mathrm{d}}^{-1}-T_{\mathrm{b}}^{-1}$, we obtain the following approximation

$$
\tau_{\mathrm{i}}>p_{\mathrm{i}} / T_{\text {cor }^{-1}}
$$

The largest lower bound is put upon $\tau_{\mathrm{j}}$ by the value of $\left(T_{1}^{-1}\right)_{\mathrm{cor}}$ from Table II. With $p_{\mathrm{i}}$ taken to be 0.04 , we find that $\tau_{\mathrm{i}}\left(\mathrm{H}_{2} \mathrm{O}\right)$ $>1 \mathrm{~ms}$, which is consistent with the 17-ms estimate for water inside human erythrocytes.

A similar analysis can be made of the exchange-averaged PRR to obtain an upper bound upon the lifetime of protons inside the thylakoids. In this case the inequality of eq 16 is reversed. When used with the values of $T_{\text {cor }}{ }^{-1}$ in Figure 3, it gives $\tau_{i}\left(\mathrm{H}^{+}\right)<20 \mathrm{~ms}$.

In summary, we conclude that the PRR of chloroplast suspensions are determined largely by the $\mathrm{Mn}$ (II) loosely bound to the inside of the membranes. Furthermore, the PRR are sensitive to changes in oxidation state of the loosely bound manganese. The effects of redox agents upon the PRR indicate that about one-fourth to one-third of the loosely bound $\mathrm{Mn}$ occurs as $\mathrm{Mn}$ (II) in dark-adapted (untreated) chloroplasts, the balance being in a higher oxidation state(s), the most likely candidate being $\mathrm{Mn}$ (III). This fraction of $\mathrm{Mn}$ (II) is comparable with the $S_{0} / S_{1}$ population ratio in the model for oxygen evolution proposed by Kok et al. (1970). A forthcoming publication will present further evidence about the relationship between the PRR and the oxygen-evolving mechanism in photosynthesis. In light-flash experiments, the PRR were found to exhibit a damped oscillation with a periodicity of four, similar to that for oxygen evolution, thereby enabling one to explore the occurrence of changes in the oxidation state of manganese during oxygen evolution.

\section{Acknowledgments}

The generous assistance of Dr. J. Jonas and Mr. G. Munie in making some of the oxygen-17 NMR measurements is gratefully acknowledged.

\section{References}

Andrasko, J. (1976), Biochim. Biophys. Acta 428, 304.

Arnon, D. I. (1949), Plant Physiol. 24, 1.

Blankenship, R. E., and Sauer, K. (1974), Biochim. Biophys. Acta 357, 252.

Bloembergen, N., and Morgan, L. O. (1961), J. Chem. Phys. 34,842 .

Blumberg, W. E., and Peisach, J. (1966), Biochim. Biophys. Acta 126, 269.

Carr, H. Y., and Purcell, E. M. (1954), Phys. Rev. 94, 630.

Case, G. D. (1975), Biochim. Biophys. Acta 375, 69
Chen, K. Y., and Wang, J. H. (1974), Bioinorg. Chem. 3, 339.

Cheniae, G. M. (1970), Annu. Rev. Plant Physiol. 21, 467.

Cheniae, G. M., and Martin, I. F. (1966), Brookhaven Symp. Biol. 19, 406.

Cheniae, G. M., and Martin, I. F. (1971), Plant Physiol. 47, 568.

Dwek, R. A. (1973), Nuclear Magnetic Resonance (N.M.R.) in Biochemistry: Application to Enzyme Systems, Oxford, Clarendon Press, Chapters 9 and 10.

Earley, J. E. (1973), Inorg. Nucl. Chem. Lett. 9, 487.

Gutowsky, H. S., Vold, R. L., and Wells, E. J. (1965), J. Chem. Phys. 43, 4107.

Henriques, F., and Park, R. B. (1976), Biochim. Biophys. Acta $430,312$.

Homann, P. H. (1968), Biochem. Biophys. Res. Commun. 33, 229.

Johri, M. M., and Varner, J. E. (1964) in Methods in Developmental Biology, Wilt, F. H., and Wessells, N. K., Ed., New York, N.Y., Thomas Y. Crowell, p 604.

Kessler, E., Arthur, W., and Brugger, J. E. (1957), Arch. Biochem. Biophys. 71, 326.

Kok, B., Forbush, B., and McGloin, M. (1970), Photochem. Photobiol. 11, 457

Lagoutte, B., and Duraton, J. (1975), FEBS Lett. 51, 21.

Lozier, R., Baginsky, M., and Butler, W. L. (1971), Photochem. Photobiol. 14, 323.

Meiboom, S., and Gill, D. (1958), Rev. Sci. Instrum. 29. 688.

Mildvan, A. S., and Cohn, M. (1970), Adv. Enzymol. 33, 1.

Navon, G. (1970), Chem. Phys, Lett. 7, 390.

Olson, J. M. (1970), Science 168, 438.

Pirson, A. (1937), Z. Botan. 31, 193.

Renger, G. (1970), Z. Naturforsch. 256, 966.

Reuben, J., and Cohn, M. (1970), J. Biol. Chem. 245, 6539.

Shporer, M., and Civan, M. M. (1975), Biochim. Biophys. Acta 385,81 .

Siderer, Y., Malkin, S., Poupko, R., and Luz, Z. (1977), Arch. Biochem. Biophys. 179, 174.

Spencer, D., and Possingham, J. V. (1961), Biochim. Biophys. Acta 52, 379.

Tel-Or, E., and Avron, M. (1974), in Proceedings of the 3rd International Photosynthesis Congress, Vol. 1, Rehovot, Israel, Avron, M., Ed., p 569.

Trebst, A. (1974), Annu. Rev. Plant Physiol. 25, 423.

Vieira, F. L., Shaafi, R. I., and Solomon, A. K. (1970), J. Gen. Physiol. 55, 451.

Villafranca, J. J., Yost, F. J., and Fridovich, I. (1974), J. Biol. Chem. 249, 3532.

Wydrzynski, T., Zumbulyadis, N., Schmidt, P. G., and Govindjee (1975), Biochim. Biophys. Acta 408, 349.

Wydrzynski, T., Zumbulyadis, N., Schmidt, P. G., Gutowsky, H. S., and Govindjee (1976a), Proc. Natl. Acad. Sci. U.S.A. $73,1196$.

Wyrdrzynski, T., Govindjee, Zumbulyadis, N., Schmidt, P. G., and Gutowsky, H. S. (1976b), ACS Symp. Ser. 34. 471.

Yamashita, T., and Butler, W. L. (1968), Plant Physiol. 43, 1978.

Yamashita, T., and Tomita, G. (1974), Plant Cell Physiol. 15, 252. 